Discrete Comput Geom 29:23-39 (2003)

DOI: $10.1007 / \mathrm{s} 00454-002-2791-7$

Geometry

\title{
Densest Packing of Equal Spheres in Hyperbolic Space*
}

\author{
Lewis Bowen ${ }^{\dagger}$ and Charles Radin \\ Mathematics Department, University of Texas at Austin, \\ Austin, TX 78712-1082, USA \\ \{lbowen,radin\}@math.utexas.edu
}

\begin{abstract}
We propose a method to analyze the density of packings of spheres of fixed radius in the hyperbolic space of any dimension $m \geq 2$, and prove that for all but countably many radii, optimally dense packings must have low symmetry.
\end{abstract}

\section{Introduction}

While the study of densest packings of spheres in Euclidean space has made impressive gains in recent years $[\mathrm{H}]$, the analogous study in hyperbolic space has been held back at a fundamental level; there has not been a convincing approach to define what one should mean by "densest packing of spheres" in hyperbolic space [Bo2], [Fej], [FeK], [FKK]; see especially the discussion on pp. 831-834 of [FeK].

Intuitively the difficulty in hyperbolic space is due to the feature that in a packing of equal spheres the ratio, of the number of spheres intersecting the surface of a region to the number contained in that region, need not vanish as the region increases in size, and therefore defining density in (the noncompact) hyperbolic space, as the limit of the relative density within expanding compact subregions, is too sensitive to the details of the boundary of the subregions. There is an instructive example by Böröczky [Bo1], $[\mathrm{FeK}]$ of a packing $x$ of congruent disks in the hyperbolic plane, together with a pair of tilings, $T_{1}$ and $T_{2}$, with the following properties. Each tiling consists of congruent copies of a single polygonal tile, and each tile in the corresponding tiling contains a single disk of $x$; but the tile for $T_{1}$ has a larger volume than that of $T_{2}$, so the "relative density" of the packing $x$ would be lower if defined using $T_{1}$ rather than $T_{2}$.

In this paper we do not attempt to define densities for all packings of hyperbolic space, for instance that of Böröczky. However, we show that there is a natural meaning

\footnotetext{
* This research was supported in part by Texas ARP Grant 003658-158 and NSF Grant DMS-0071643.

$\dagger$ Current address: Mathematics Department, University of California at Davis, Davis, CA 95616, USA. lbowen@math.ucdavis.edu.
} 
for "optimally dense" packings in hyperbolic space. Furthermore, we show that only for a special countable set of radius values $R$ do congruent balls of radius $R$ have optimally dense packings with high symmetry. This contrasts with Euclidean space for dimensions 2 and 3, where for each radius $R$ there exists a high symmetry packing (lattice packing) of spheres with radius $R$ which is optimally dense. Intuitively, to define "optimally dense packings" we associate to a packing $x$ an invariant measure $\mu_{x}$ on a space of packings and define an average density $D\left(\mu_{x}\right)$ to such a measure. It should be noted, however, that we cannot do this for every packing since there are packings like Börözcky's example that have no well-defined density. So our methods necessarily entail a restriction on the kinds of packings under consideration. This may seem unexpected from the classical viewpoint. In the conclusion of the paper we justify the appropriateness of our mathematical approach in defining optimal density packings.

\section{Definitions and Results}

For definiteness we choose a metric $d(\cdot, \cdot)$ such that our $m$-dimensional hyperbolic space $\mathbb{H}^{m}, m \geq 2$, has curvature -1 , and choose a distinguished "origin" $\mathcal{O}$ so we can identify $\mathbb{H}^{m}$ with the space $\mathcal{G} / \Sigma_{\mathcal{O}}$ of left cosets of the orientation-preserving unimodular isometry group $\mathcal{G}$ of $\mathbb{H}^{m}$ by the (compact) stabilizer subgroup $\Sigma_{\mathcal{O}}$ of $\mathcal{O}$, and $\mathcal{G}$ acts on $\mathbb{H}^{m}$ on the left. (See $[\mathrm{V}]$ for a general reference on hyperbolic spaces and their congruence groups.)

We note that if $\mathcal{G}$ has its usual metrizable topology and $\mathcal{G} / \Sigma_{\mathcal{O}}$ its quotient topology, the pairing, of the left cosets $g \Sigma_{\mathcal{O}}$ with the images of $\mathcal{O}$ under $g$, is a homeomorphism. We fix the normalization of the Haar measure $\mu_{\mathcal{G}}$ on $\mathcal{G}$ such that its connection with the measure $\mu_{m}$ on $\mathbb{H}^{m}$ associated with its metric is $\mu_{m}[S]=\mu_{\mathcal{G}}\left[\pi_{\mathcal{O}}^{-1}(S)\right]$, where $\pi_{\mathcal{O}}$ is the projection of $\mathcal{G}$ onto $\mathcal{G} / \Sigma_{\mathcal{O}}$.

We are concerned with the density of packings of $\mathbb{H}^{m}$ by congruent closed balls. By a closed ball (of radius $R$ ) in a metric space we mean the set of points in the space whose distance from some point—called the "center"—is less than or equal to $R$. By a packing of a metric space by balls we mean a collection of balls in that space with pairwise disjoint interiors.

In Euclidean space one can show, using similarity, that the maximal density of packings by congruent balls is independent of the radius of the balls. In hyperbolic space this is not the case, as we note at the end of this section. In part to analyze this, we consider the symmetry of packings.

\section{Definition 1.}

(a) The "symmetry group" of packing $x$ is the group $\Gamma_{x}=\{g \in \mathcal{G} \mid g x=x\}$, using the notation $g x$ to denote the natural action of $g \in \mathcal{G}$ on $x$ by the rigid motion $g$.

(b) A packing for which the symmetry group is cocompact in $\mathcal{G}$ is called a "periodic packing." It is a "nonperiodic packing" otherwise.

For all dimensions we use the following notation introduced in [Bow] (where packings with such balls were studied). 
Definition 2. The radius $R$ of a ball in $\mathbb{H}^{m}$ is "tight" if the regular simplex of side length $2 R$ admits a (full-face to full-face) tiling of $\mathbb{H}^{m}$, called the associated "tightsimplex packing."

For balls of a fixed tight radius $R$, there is a canonical packing with the centers of the balls placed at the vertices of the simplices. It can be shown that this packing has high symmetry: it has a symmetry group, cocompact in $\mathcal{G}$, with a fundamental domain that can be fashioned from part of the simplex determined by the centers of $m+1$ balls which are pairwise neighbors, that is, have centers separated by $2 R$. We show that this packing is optimally dense according to the definition we introduce below.

Our objective is to define a notion of optimally dense packings for balls of radius $R>0$. We analyze the situation for the whole range of positive radii, and show that, in contrast to the case of tight radii, for most radii the optimally dense packings cannot have a symmetry group with compact fundamental domain, or, equivalently [Bea], [Kat], cannot have a symmetry group which is cocompact in the isometry group $\mathcal{G}$.

We use the following notation, consistent for instance with [MaM].

Definition 3. A packing for which the symmetry group is cocompact in $\mathcal{G}$ is called "periodic" or said to have "high" symmetry. The remaining packings are called "nonperiodic" and said to have "low" symmetry.

Our next objective is a definition for optimally dense packings. For fixed $R>0$ we begin with the space $S_{R}$ of all "relatively dense" packings of $\mathbb{H}^{m}$ by (closed) $R$-balls, that is, packings $x$ of $\mathbb{H}^{m}$, with $R$-balls, having the property that any closed $R$-ball in $\mathbb{H}^{m}$ intersects a ball of $x$. For a packing $x$ we denote the set of centers of its balls by $C_{x}$. On $S_{R}$ we put the following metric:

$$
d_{R}(x, y)=\sup _{n \geq 1} \frac{1}{n} h\left(B_{n} \cap C_{x}, B_{n} \cap C_{y}\right),
$$

where $B_{n}$ denotes the closed ball of radius $n$ centered at the origin, and for compact sets $A$ and $B$ we use the Hausdorff metric

$$
h(A, B) \equiv \max \left\{\sup _{a \in A} \inf _{b \in B} d(a, b), \sup _{b \in B} \inf _{a \in A} d(a, b)\right\} .
$$

It is not hard to see [RaW] that $S_{R}$ is compact in this metric topology, and that the natural action $(g, x) \in \mathcal{G} \times S_{R} \longrightarrow g(x) \in S_{R}$ of the isometry group $\mathcal{G}$ of $\mathbb{H}^{m}$ on $S_{R}$ is (jointly) continuous. Let $\mathcal{M}(R)$ be the family of Borel probability measures on $S_{R}$. We call a measure $\mu \in \mathcal{M}(R)$ "invariant" if for any Borel set $E \in S_{R}$ and any $g \in \mathcal{G}$, $\mu(g E)=\mu(E)$. Let $\mathcal{M}_{I}(R)$ be the subset of invariant measures and let $\mathcal{M}_{I}^{\mathrm{e}}(R)$ be the convex extreme ("ergodic") points of $\mathcal{M}_{I}(R)$, all in their weak* topology, in which $\mathcal{M}(R)$ and $\mathcal{M}_{I}(R)$ are compact.

Some relevant elements of $\mathcal{M}_{I}^{\mathrm{e}}(R)$ can be constructed as follows. Suppose $x$ is a periodic packing, i.e. the symmetry group $\Gamma_{x}$ of $x$ is cocompact in $\mathcal{G}$. We will construct a measure $\mu_{x} \in \mathcal{M}_{I}^{\mathrm{e}}(R)$ whose support is contained in the orbit $O(x) \equiv\{g x \mid g \in$ $\mathcal{G}\} \subset S_{R}$ of $x . O(x)$ is naturally homeomorphic to the (metrizable) space $\mathcal{G} / \Gamma_{x}$ of 
left cosets by the homeomorphism $q_{x}: O(x) \rightarrow \mathcal{G} / \Gamma_{x}$ with $q_{x}(g x)=g \Gamma_{x}$. There is a natural probability measure on $\mathcal{G} / \Gamma_{x}$ induced by Haar measure on $\mathcal{G}$ by the projection map $\pi_{x}: \mathcal{G} \rightarrow \mathcal{G} / \Gamma_{x}$. (Aside from an overall normalization the measure on $\mathcal{G} / \Gamma_{x}$ can be defined on sufficiently small open balls $B \subset \mathcal{G} / \Gamma_{x}$ as the Haar measure of any of the components of $\pi_{x}^{-1}(B)$.) Hence $q_{x}$ induces a probability measure $\hat{\mu}_{x}$ on $O(x)$. This measure can then be extended to all of $S_{R}$ in the following way: $\mu_{x}(E)=\hat{\mu}_{x}(E \cap O(x))$ for any Borel set $E \subseteq S_{R}$. We use the term "periodic measure" to denote any measure in $M_{I}(R)$ associated in this way with the orbit of a periodic packing. It is not hard to prove from the uniqueness of the Haar measure on $\mathcal{G}$ that there is only one probability measure, with support in the orbit of a periodic packing, which is invariant under $\mathcal{G}$.

For any $p \in \mathbb{H}^{m}$ we now define the real-valued function $F_{p}$ on $S_{R}$ as the indicator function of the set of all packings $x$ such that $p$ is contained in a ball of $x$. (The latter condition will sometimes be expressed as $p \in x$.) Finally, in the spirit of [BeS] we define optimal density through invariant measures as follows.

Definition 4. For any invariant measure $\mu \in \mathcal{M}_{I}(R)$, the "average density" $D(\mu)$ is defined as $\int_{S_{R}} F_{p}(y) d \mu(y)$.

Note: the average density $D(\mu)$ is independent of the choice of $p$, because of the invariance of the measure, so $p$ is not needed in the notation. For convenience we sometimes use $p=\mathcal{O}$.

For periodic packings $x$ there is an obvious notion of density using a fundamental domain of $\Gamma_{x}$. We prove that our definition of density coincides with this intuitive notion for such special $x$.

Proposition 1. If $x$ is a periodic packing, $D\left(\mu_{x}\right)$ is the relative volume of any fundamental domain for $\Gamma_{x}$ taken up by the balls of $x$.

Another justification for this notion of density comes from an ergodic theorem of Nevo ([Ne, Theorem 1] for dimension $m \geq 3$; [NeS, Theorem 3] for $m \geq 2$ ) which has the following consequence.

Proposition 2. For any ergodic measure $\mu \in \mathcal{M}_{I}^{\mathrm{e}}(R)$, and $\mu$-almost every packing $y \in S_{R}$, the average density $D(\mu)$ is the limit of the relative fraction in expanding spheres centered at $\mathcal{O}$ taken up by the balls in the packing $y$.

We now define optimality through measures.

Definition 5. $\quad D(R) \equiv \sup _{\mu \in \mathcal{M}_{I}^{\mathrm{e}}(R)} D(\mu)$ is called the "optimal density for radius $R$," and any ergodic measure $\tilde{\mu} \in \mathcal{M}_{I}^{\mathrm{e}}(R)$ is called "optimally dense (for radius $R$ )" if $D(\tilde{\mu})=D(R)$. Those packings, in the support of an optimally dense measure, whose orbit under $\mathcal{G}$ is dense in the support of that measure, are called "optimally dense" (for that radius).

Our first main result asserts the existence of optimally dense packings. 
Theorem 1. For any radius $R>0$ there exists an optimally dense measure $\mu \in$ $\mathcal{M}_{I}^{\mathrm{e}}(R)$, and a subset of the support of $\mu$, of full $\mu$-measure, of optimally dense packings.

There may be many optimally dense measures for a given $R$.

We next consider the question of the existence of optimally dense packings having high symmetry. We prove the following result.

Theorem 2. There are only countably many radii $R>0$ for which there exist periodic optimally dense packings. Balls with tight radii have periodic optimally dense packings.

This result shows that for most values of $R$ every optimal packing is nonperiodic, that is, has low symmetry.

We use the following examples of packings with tight radii. In the hyperbolic plane there are equilateral triangles with any positive angle less than $\pi / 3$, and furthermore the side length of an equilateral triangle is determined by the angle. If $R_{n}$ is half the side length of an equilateral triangle of angle $2 \pi / n$ for some integer $n>6$ (i.e. $\cosh \left(2 R_{n}\right)=$ $\cot (2 \pi / n) \cot (\pi / n))$, then about a "central" disk of radius $R_{n}$ in the plane one can position $n$ other disks of that radius, all having centers at distance $2 R_{n}$ from that of the central disk, and with the interiors of the $n+1$ disks being pairwise disjoint. For each $n$ this extends naturally to what we called above a tight-simplex packing; the "density" of such a packing, which we show by Theorem 2 to be optimal, is easily computed to be $[3 \csc (\pi / n)-6] /[n-6]$ (see p. 239 of [Fej]), and is seen to be increasing in $n$.

\section{Computing Optimal Density}

We begin with a proof of Proposition 1, which deals with the simplest sort of packings, those which are periodic.

Proof of Proposition 1. Suppose $x$ is a periodic packing. Let $\mathcal{F} \subset \mathbb{H}^{m}$ be a fundamental domain for $\Gamma_{x}$, let $\hat{\mathcal{F}}=\pi_{\mathcal{O}}^{-1} \mathcal{F}$, let $K_{x} \subset \mathbb{H}^{m}$ be the union of the disks in $x$ and let $K_{\mathcal{O}} \subset S_{R}$ be the set of all packings for which $\mathcal{O}$ is in a disk. Recall that we associated to a periodic packing $x$ a measure $\mu_{x}$ on $S_{R}$ constructed using projection maps $\pi_{x}: \mathcal{G} \rightarrow$ $\mathcal{G} / \Gamma_{x}$ and an isomorphism $q_{x}: O(x) \rightarrow \mathcal{G} / \Gamma_{x}$. It is not hard to see that $\pi_{\mathcal{O}}^{-1}\left[K_{x}\right]=$ $\pi_{x}^{-1}\left\{q_{x}\left[K_{\mathcal{O}} \cap q_{x}^{-1}\left(\mathcal{G} / \Gamma_{x}\right)\right]\right\}$ and therefore

$$
\begin{aligned}
\frac{\mu_{m}\left(K_{x} \cap \mathcal{F}\right)}{\mu_{m}(\mathcal{F})} & =\frac{\mu_{\mathcal{G}}\left(\pi_{\mathcal{O}}^{-1}\left[K_{x}\right] \cap \hat{\mathcal{F}}\right)}{\mu_{\mathcal{G}}(\hat{\mathcal{F}})}=\frac{\mu_{\mathcal{G}}\left(\pi_{x}^{-1}\left\{q_{x}\left[K_{\mathcal{O}} \cap q_{x}^{-1}\left(\mathcal{G} / \Gamma_{x}\right)\right]\right\} \cap \hat{\mathcal{F}}\right)}{\mu_{\mathcal{G}}(\hat{\mathcal{F}})} \\
& =\hat{\mu}_{x}\left\{q_{x}\left[K_{\mathcal{O}} \cap q_{x}^{-1}\left(\mathcal{G} / \Gamma_{x}\right)\right]\right\}=\mu_{x}\left(K_{\mathcal{O}}\right)
\end{aligned}
$$

which proves the proposition.

Proof of Propostion 2. Applying Nevo's theorem [NeS, Theorem 3] to the indicator function $F_{\mathcal{O}}$, we get that $D(\mu)=\int_{X} F_{\mathcal{O}} d \mu$ can be computed as the limit $\lim _{n \rightarrow \infty}\left[1 / \mu_{\mathcal{G}}\left(\pi_{\mathcal{O}}^{-1} B_{n}\right)\right] \int_{\pi_{\mathcal{O}}^{-1} B_{n}} f(g z) d \mu_{\mathcal{G}}(g)$. The connection between the invariant 


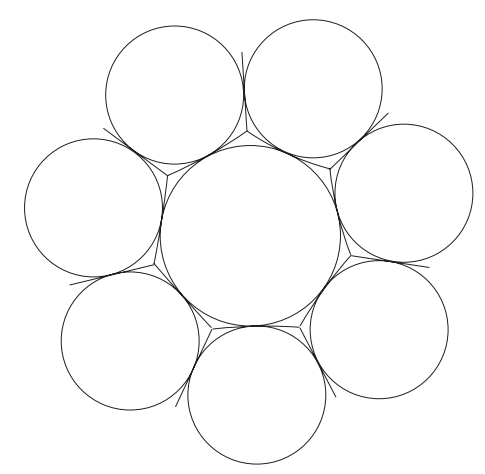

Fig. 1. Voronoi cell, radius $R_{7}$.

measures on $\mathcal{G}$ and $\mathbb{H}^{m}$, as noted at the beginning of this section, then implies that for $\mu$-almost every packing $x \in S_{R}, D(\mu)=\lim _{n \rightarrow \infty}\left[1 / \mu_{m}\left(B_{n}\right)\right] \int_{B_{n}} F_{q}(x) d \mu_{m}(q)$, which proves the proposition.

For $y \in S_{R}$ we wish to focus on two ways to represent the packing in terms of tilings of $\mathbb{H}^{m}$. For the first, consider, for each ball center $a \in C_{y}$, the geodesic segment $a b$ which joins $a$ to any $b \in C_{y}, b \neq a$, and the two half-spaces defined by the geodesic hyperplane which is the perpendicular bisector of $a b$. Let $\mathcal{B}(a)$ be the subset of these half-spaces in which $a$ is contained. The "Voronoi cell of $a$," which clearly contains the ball centered at $a$, is the closure of the intersection of the half-spaces in $\mathcal{B}(a)$. The Voronoi cells of $y$ form a tiling of $\mathbb{H}^{m}$, which we call the "Voronoi tiling of $y$," the first of the tilings of $\mathbb{H}^{m}$ associated with $y$ with which we are interested. In a tight-simplex packing the spheres constitute a single orbit, under the corresponding symmetry group, of any one of the spheres. So in particular all the spheres have congruent Voronoi cells. We illustrate this for dimension 2, with a disk of a tight-simplex packing sitting in its Voronoi cell; Fig. 1 (resp. Fig. 2) corresponds to radius $R_{7}$, which is approximately 0.6391 (resp. $R_{8}$, which is approximately 0.8074 ), with density approximately 0.9143 (resp. 0.9197). Both figures are in the Poincaré disk model (see [Rat]).

To define the other tiling of $\mathbb{H}^{m}$ associated with $y$, consider any ball $B$ whose interior does not contain any point of $C_{y}$ and whose boundary intersects $C_{y}$ in a set not contained in any proper geodesic hyperplane of $\mathbb{H}^{m}$. The convex hull of $B \cap C_{y}$ is called a "Delone cell" (or "dual Voronoi cell") of $y$.

For those $y \in S_{R}$ such that $\mathcal{O}$ is contained in the interior of a Voronoi cell (which cell we denote by $V_{\mathcal{O}}(y)$ ), we define $\tilde{F}(y)$ to be the relative volume of $V_{\mathcal{O}}(y)$ occupied by the balls of $y$. (We note that $\tilde{F}(y)$ is defined $\mu$-almost everywhere for any $\mu \in \mathcal{M}_{I}(R)$.)

Definition 6. For invariant measures $\mu \in \mathcal{M}_{I}(R)$ we define the "average Voronoi density for $\mu, " D_{V}(\mu)$, as $\int_{S_{R}} \tilde{F}(y) d \mu(y)$.

Similarly, for $y \in S_{R}$ such that $\mathcal{O}$ is contained in the interior of a Delone cell, we define $\hat{F}(y)$ as the relative volume of the Delone cell of $y$ that contains the origin occupied by the balls of $y$. (We note that $\hat{F}(y)$ is defined $\mu$-almost everywhere for any $\mu \in \mathcal{M}_{I}(R)$.) 


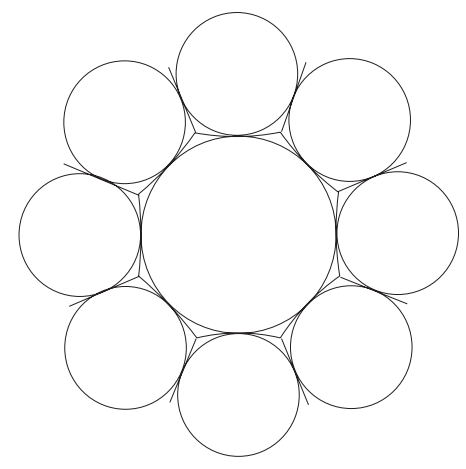

Fig. 2. Voronoi cell, radius $R_{8}$.

Definition 7. For invariant measures $\mu \in \mathcal{M}_{I}(R)$ we define the "average Delone density for $\mu, " D_{\hat{V}}(\mu)$, as $\int_{S_{R}} \hat{F}(y) d \mu(y)$.

Proposition 3. For any invariant measure $\mu \in \mathcal{M}_{I}(R)$, the average Voronoi density $D_{V}(\mu)$ and the average Delone density $D_{\hat{V}}(\mu)$ both equal the average density $D(\mu)$.

Proof. We prove that $D_{V}(\mu)=D(\mu)$; the case for the Delone density follows similarly. For any subset $X$ of $\mathbb{H}^{m}$ and any $r>0$ let $N_{r}(X)$ denote the open $r$-neighborhood of $X$, that is, the union of all open $r$-balls centered at points of $X$. For a closed subset $X \subset \mathbb{H}^{m}$, let $\partial X$ denote the intersection of $X$ with the closure of the complement of $X$.

Let $\mathcal{V}$ be the set of all Voronoi cells $V_{\mathcal{O}}(x)$ of all packings $x$ such that $\mathcal{O} \in C_{x}$. Define a metric $d_{\mathcal{V}}$ on $\mathcal{V}$ by the following:

$$
d_{V}(v, w)=\inf \left\{\varepsilon>0 \mid \partial w \subset N_{\varepsilon}(\partial v) \text { and } \partial v \subset N_{\varepsilon}(\partial w)\right\} .
$$

It is easy to check that $\mathcal{V}$ is compact under this metric.

Let $\varepsilon>0$ be given. We choose $\delta>0$ such that the set $\left\{x \in S_{R} \mid \mathcal{O} \in N_{\delta}\left(\partial V_{\mathcal{O}}(x)\right)\right\}$ has $\mu$-measure less than $\varepsilon$. We also require that for any $v \in \mathcal{V}$,

$$
\left|\frac{\mu_{m}\left(B_{R}\right)}{\mu_{m}\left(v-N_{\delta}(\partial v)\right)}-\frac{\mu_{m}\left(B_{R}\right)}{\mu_{m}(v)}\right|<\varepsilon
$$

A $\delta>0$ exists with the above properties because of our assumption that all packings $x \in S_{R}$ are relatively dense and because $\mu$ is invariant.

Since $\mathcal{V}$ is compact there exists a finite collection $\left\{V_{j}\right\}_{j=1}^{n}$ of subsets of $\mathcal{V}$ such that

(a) $V_{j} \cap V_{k}=\emptyset$ for $j \neq k$;

(b) $\bigcup_{j} V_{j}=\mathcal{V}$;

(c) $V_{j}$ is Borel for all $j$;

(d) if $v \in V_{j}, w \in V_{k}, g \in \Sigma_{\mathcal{O}}$ and $g v=w$, then $j=k$;

(e) for any $j$, and for all $v$ and $w$ in $V_{j},\left|\mu_{m}\left(B_{R}\right) / \mu_{m}(v)-\mu_{m}\left(B_{R}\right) / \mu_{m}(w)\right|<\varepsilon$;

(f) if $v, w \in V_{j}$, then there exists a $h \in \Sigma_{\mathcal{O}}$ so that $d_{\mathcal{V}}(h v, w)<\delta / 2$ and $G_{h v}=$ $\{g \in \mathcal{G} \mid g h v=v\}=G_{w}=\{g \in \mathcal{G} \mid g w=w\}$, i.e. $h v$ and $w$ have the same symmetry group. 
For each $j>0$, fix an element $v_{j}$ of $V_{j}$ and choose a Borel set $V_{j}^{\prime} \subset V_{j}$ such that

(i) for each $v \in V_{j}$ there exists a $g \in \Sigma_{\mathcal{O}}$ such that $g v \in V_{j}^{\prime}$;

(ii) if $v \in V_{j}$ and $g_{1}, g_{2} \in \Sigma_{\mathcal{O}}$ and $g_{1} v, g_{2} v \in V_{j}^{\prime}$, then $g_{1} v=g_{2} v$, i.e. $g_{1}^{-1} g_{2}$ is in the symmetry group of $v$;

(iii) for each $v \in V_{j}^{\prime}, G_{v}=G_{v_{j}}$;

(iv) for each $v \in V_{j}^{\prime}, d_{\mathcal{V}}\left(v, v_{j}\right)<\delta / 2$.

For each $j>0$, let $U_{j}=\bigcap_{v \in V_{j}^{\prime}} v$. Then each point $p \in \partial U_{j}$ is in $\partial w$ for some $w \in V_{j}^{\prime}$. Hence by the fourth condition on $V_{j}^{\prime}, \partial U_{j} \subset N_{\delta / 2}\left(\partial v_{j}\right)$. Again by the fourth condition, this implies that $\partial U_{j} \subset N_{\delta}(\partial v)$ for all $v \in V_{j}^{\prime}$. Note also that $v_{j}-N_{\delta}\left(\partial v_{j}\right) \subset U_{j}$.

For each $j>0$ and any Borel set $U \subset \mathbb{H}^{m}$, define $C_{j}(U)=\left\{x \in S_{R} \mid\right.$ there exists $g \in \mathcal{G}$ such that $\mathcal{O} \in g U, g \mathcal{O} \in C_{x}$ and $\left.g^{-1} V_{\mathcal{O}}(x) \in V_{j}^{\prime}\right\}$.

To finish the proof, we prove each of the following (in-)equalities in order:

$$
\begin{aligned}
\left|D(\mu)-D_{V}(\mu)\right| & =\left|\int_{S_{R}} F_{\mathcal{O}}-\tilde{F} d \mu\right| \\
& \leq\left|\int_{\cup_{j} C_{j}\left(U_{j}\right)} F_{\mathcal{O}}-\tilde{F} d \mu\right|+\varepsilon \\
& =\left|\Sigma_{j} \int_{C_{j}\left(U_{j}\right)} F_{\mathcal{O}}-\tilde{F} d \mu\right|+\varepsilon \\
& =\left|\Sigma_{j} \mu\left(C_{j}\left(B_{R}\right)\right)-\int_{C_{j}\left(U_{j}\right)} \tilde{F} d \mu\right|+\varepsilon \\
& =\left|\Sigma_{j}\left(\frac{\mu_{m}\left(B_{R}\right)}{\mu_{m}\left(U_{j}\right)}\right) \mu\left(C_{j}\left(U_{j}\right)\right)-\int_{C_{j}\left(U_{j}\right)} \tilde{F} d \mu\right|+\varepsilon \\
& =\left|\Sigma_{j} \int_{C_{j}\left(U_{j}\right)} \frac{\mu_{m}\left(B_{R}\right)}{\mu_{m}\left(U_{j}\right)}-\tilde{F} d \mu\right|+\varepsilon \\
& \leq \Sigma_{j} \int_{C_{j}\left(U_{j}\right)}\left|\frac{\mu_{m}\left(B_{R}\right)}{\mu_{m}\left(U_{j}\right)}-\tilde{F}\right| d \mu+\varepsilon \\
& \leq \Sigma_{j} \int_{C_{j}\left(U_{j}\right)}\left|\frac{\mu_{m}\left(B_{R}\right)}{\mu_{m}\left(v_{j}\right)}-\tilde{F}\right| d \mu+2 \varepsilon \\
& \leq 3 \varepsilon
\end{aligned}
$$

Equation (5) holds by definition. Equation (6) will follow once we prove that $\mu\left(S_{R}-\bigcup_{j} C_{j}\left(U_{j}\right)\right)<\varepsilon$. By the choice of $\delta$, it suffices to prove that $S_{R}-\bigcup_{j} C_{j}\left(U_{j}\right) \subset$ $\left\{x \in S_{R} \mid \mathcal{O} \in N_{\delta}\left(\partial V_{\mathcal{O}}(x)\right)\right\}$. So let $x \in S_{R}-\bigcup_{j} C_{j}\left(U_{j}\right)$. Let $g \in \mathcal{G}$ and $j>0$ be such that $g^{-1} V_{\mathcal{O}}(x) \in V_{j}^{\prime}$ and $g \mathcal{O} \in C_{x}$. Then $\mathcal{O} \notin g U_{j}$ since $x \notin C_{j}\left(U_{j}\right)$. So $g^{-1} \mathcal{O} \in g^{-1} V_{\mathcal{O}}(x) \in V_{j}^{\prime}$ but $g^{-1} \mathcal{O} \notin U_{j}$. From the discussion after the definition of $U_{j}$ it follows that $g^{-1} \mathcal{O} \in N_{\delta}\left(\partial g^{-1} V_{\mathcal{O}}(x)\right)$ which implies $\mathcal{O} \in N_{\delta}\left(\partial V_{\mathcal{O}}(x)\right)$ as required. 
For (7), it suffices to prove that whenever $j \neq k, C_{j}\left(U_{j}\right) \cap C_{k}\left(U_{k}\right)=\emptyset$. So suppose $x \in C_{j}\left(U_{j}\right) \cap C_{k}\left(U_{k}\right)$. Then there exists $g_{j}, g_{k} \in \mathcal{G}$ such that $\mathcal{O} \in g_{j} U_{j} \cap g_{k} U_{k}$, $g_{j} \mathcal{O}, g_{k} \mathcal{O} \in C_{x}$ and $g_{j}^{-1} V_{\mathcal{O}}(x)$ and $g_{k}^{-1} V_{O}(x)$ are in $V_{j}^{\prime}$. Since $g_{j} \mathcal{O}$ and $g_{k} \mathcal{O}$ are in $C_{x}$ and in $V_{\mathcal{O}}(x)$ it must be that $g_{j} \mathcal{O}=g_{k} \mathcal{O}$. Hence $g_{k}^{-1} g_{j} \in \Sigma_{\mathcal{O}}$. Since there exists $w_{j} \in V_{j}^{\prime}$ and $w_{k} \in V_{j}^{\prime}$ such that $g_{j} w_{j}=V_{\mathcal{O}}(x)=g_{k} w_{k}, g_{k}^{-1} g_{j} w_{j}=w_{k}$. Now condition (d) on the collection $\left\{V_{i}\right\}_{i=1}^{n}$ implies that $j=k$ as required.

For (8), it suffices to show that $\int_{C_{j}\left(U_{j}\right)} F_{\mathcal{O}}=\mu\left(C_{j}\left(B_{R}\right)\right)$. Note that $F_{\mathcal{O}}$ is the characteristic function of the set $K=\left\{x \in S_{R} \mid \mathcal{O} \in x\right\}$. Hence it suffices to prove that $\mu\left(C_{j}\left(U_{j}\right) \cap K\right)=\mu\left(C_{j}\left(B_{R}\right)\right)$. We show that $C_{j}\left(U_{j}\right) \cap K=C_{j}\left(B_{R}\right)$. So let $x \in C_{j}\left(U_{j}\right) \cap K$. Let $g \in \mathcal{G}$ and $w \in V_{j}^{\prime}$ such that $\mathcal{O} \in g U_{j}, g \mathcal{O} \in C_{x}$ and $g w=V_{\mathcal{O}}(x)$. Since $x \in K, \mathcal{O}$ is contained in the ball of $x$ that is contained in $V_{\mathcal{O}}(x)$. Since $g w=V_{\mathcal{O}}(x)$ and $g \mathcal{O} \in C_{x}$, this ball is equal to $g B_{R}$. So $\mathcal{O} \in g B_{R}$ which implies that $x \in C_{j}\left(B_{R}\right)$. Since $x$ is arbitrary, $C_{j}\left(U_{j}\right) \cap K \subset C_{j}\left(B_{R}\right)$.

Now let $x \in C_{j}\left(B_{R}\right)$. It is clear from the definitions that $x \in C_{j}\left(U_{j}\right)$. Let $g \in \mathcal{G}$ such that $\mathcal{O} \in g B_{R}$ and $g \mathcal{O} \in C_{x}$. Since $\mathcal{O} \in g B_{R}, d(\mathcal{O}, g \mathcal{O}) \leq R$. Since $g \mathcal{O} \in C_{x}$, this implies that $d\left(\mathcal{O}, C_{x}\right) \leq R$, i.e. $\mathcal{O} \in x, x \in K$. Since $x$ is arbitrary, $C_{j}\left(B_{R}\right) \subset C_{j}\left(U_{j}\right) \cap K$ as required.

Next we prove (9). It suffices to prove that $\mu\left(C_{j}\left(U_{j}\right)\right)=\mu\left(C_{j}\left(B_{R}\right)\right)\left(\mu_{m}\left(U_{j}\right) /\right.$ $\mu_{m}\left(B_{R}\right)$ ). First we define a function $\Psi: C_{j}\left(U_{j}\right) \rightarrow \mathcal{G}$ (we denote $\Psi(x)$ by $\Psi_{x}$ ). This function will soon be used to define a (left) invariant measure on $\mathcal{G}$. For each $j>0$, let $\mathcal{F}_{j}$ be the interior of a fundamental domain for the symmetry group $G_{v_{j}}$ of $v_{j}$. We show that for every $x \in C_{j}\left(U_{j}\right)$ there is a unique $g \in \mathcal{G}$ such that $\mathcal{O} \in g\left(U_{j} \cap \mathcal{F}_{j}\right), g \mathcal{O} \in C_{x}$ and $g^{-1} V_{\mathcal{O}}(x) \in V_{j}^{\prime}$. Given this, we define $\Psi_{x}=g$.

By the definition of $C_{j}\left(U_{j}\right)$, there exists a $g \in \mathcal{G}$ such that $\mathcal{O} \in g U_{j}, g \mathcal{O} \in C_{x}$ and $g^{-1} V_{\mathcal{O}}(x) \in V_{j}^{\prime}$. By right multiplying $g$ by an element of the symmetry group of $v_{j}$ if necessary, we may assume that $\mathcal{O} \in g\left(U_{j} \cap \mathcal{F}_{j}\right)$. Now suppose that $g_{1}$ and $g_{2}$ in $\mathcal{G}$ are such that for $k=1,2, \mathcal{O} \in g_{k}\left(U_{j} \cap \mathcal{F}_{j}\right), g_{k} \mathcal{O} \in C_{x}$ and $g_{k}^{-1} V_{\mathcal{O}}(x) \in V_{j}^{\prime}$. Since there is only one element of $C_{x}$ in $V_{\mathcal{O}}(x)$ and $g_{1} \mathcal{O}$ and $g_{2} \mathcal{O}$ are in $C_{x}$ and in $V_{\mathcal{O}}(x)$, it must be that $g_{1} \mathcal{O}=g_{2} \mathcal{O}$. Hence $g_{2}^{-1} g_{1} \in \Sigma_{\mathcal{O}}$. Since $g_{1}^{-1} V_{\mathcal{O}}(x)$ and $g_{2}^{-1} V_{\mathcal{O}}(x)$ are in $V_{j}^{\prime}$, there exists $w_{1}, w_{2} \in V_{j}^{\prime}$ such that $V_{\mathcal{O}}(x)=g_{1} w_{1}=g_{2} w_{2}$. So, $g_{2}^{-1} g_{1} w_{1}=w_{2}$. Since $g_{2}^{-1} g_{1} \in \Sigma_{\mathcal{O}}$, the second condition on $V_{j}^{\prime}$ implies that $w_{1}=w_{2}$ and $g_{2}^{-1} g_{1} \in G_{w_{1}}$. The third condition on $V_{j}^{\prime}$ implies that $G_{w_{1}}=G_{v_{j}}$, so $g_{2}^{-1} g_{1} \in G_{v_{j}}$. Since $\mathcal{O} \in g_{1}\left(U_{j} \cap \mathcal{F}_{j}\right) \cap g_{2}\left(U_{j} \cap \mathcal{F}_{j}\right)$, $g_{1}^{-1} \mathcal{O} \in U_{j} \cap \mathcal{F}_{j} \cap g_{1}^{-1} g_{2}\left(U_{j} \cap \mathcal{F}_{j}\right)$. Hence $\mathcal{F}_{j} \cap g_{1}^{-1} g_{2} \mathcal{F}_{j} \neq \emptyset$ which implies that $g_{1}^{-1} g_{2}$ is the identity (since $\mathcal{F}_{j}$ in the interior of a fundamental domain $G_{v_{j}}$ ). This shows that $\Psi_{x}$ is well-defined.

We claim that if $x \in C_{j}\left(U_{j}\right)$ and $g \in \mathcal{G}$ such that $\mathcal{O} \in g \Psi_{x}\left(U_{j} \cap \mathcal{F}_{j}\right)$, then $\Psi_{g x}=g \Psi_{x}$. By definition of $\Psi, \mathcal{O} \in \Psi_{x}\left(U_{j} \cap \mathcal{F}_{j}\right), \Psi_{x} \mathcal{O} \in C_{x}$ and $\Psi_{x}^{-1} V_{\mathcal{O}}(x) \in V_{j}^{\prime}$. Hence $g \Psi_{x} \mathcal{O} \in g C_{x}=C_{g x}$ and $\left(\Psi_{x}^{-1} g^{-1}\right) g V_{\mathcal{O}}(x) \in V_{j}^{\prime}$. Since $\mathcal{O} \in g \Psi_{x}\left(U_{j} \cap \mathcal{F}_{j}\right) \subset$ $g \Psi_{x}\left[\Psi_{x}^{-1} V_{\mathcal{O}}(x)\right]=g V_{\mathcal{O}}(x)$, it must be that $g V_{\mathcal{O}}(x)=V_{\mathcal{O}}(g x)$. Hence $\Psi_{x}^{-1} g^{-1} V_{\mathcal{O}}(g x)$ $\in V_{j}^{\prime}$. It follows that $\Psi_{g x}=g \Psi_{x}$ as claimed. It is now elementary to prove that $h \Psi^{-1}(g)=$ $\Psi^{-1}(h g)$ for any $h, g \in \mathcal{G}$ satisfying $h \Psi^{-1}(g) \in C_{j}\left(U_{j}\right)$ and $g \in \Psi\left(C_{j}\left(U_{j}\right)\right)$.

Now we define a measure $\mu_{\mathcal{G}}^{\prime}$ on $\mathcal{G}$ as follows. First, if $E \subset \mathcal{G}, g \in \mathcal{G}$ and $g E \subset$ $\Psi\left(C_{j}\left(U_{j}\right)\right)$, then define $\mu_{\mathcal{G}}^{\prime}(E)=\mu\left(\Psi^{-1}(g E)\right)$. By the above $\mu_{\mathcal{G}}^{\prime}(E)$ is well-defined. For an arbitrary Borel subset $E$ of $\mathcal{G}$, define $\mu_{\mathcal{G}}^{\prime}(E)=\Sigma_{i=1}^{\infty} \mu_{\mathcal{G}}^{\prime}\left(E_{i}\right)$ where $\left\{E_{i}\right\}$ is a 
partition of $E$ for which there exists $g_{i} \in \mathcal{G}$ with $g_{i}\left(E_{i}\right) \subset \Psi\left(C_{j}\left(U_{j}\right)\right)$. Such partitions exist because $\Psi\left(C_{j}\left(U_{j}\right)\right)=\left\{g \in \mathcal{G} \mid \mathcal{O} \in g\left(U_{j} \cap \mathcal{F}_{j}\right)\right\}=\pi_{x}^{-1}\left(U_{j} \cap \mathcal{F}_{j}\right)^{-1}$ contains an open subset of $\mathcal{G}$.

Since $\mu_{\mathcal{G}}^{\prime}$ is left $\mathcal{G}$-invariant, it must be a multiple of the Haar measure $\mu_{\mathcal{G}}$ of $\mathcal{G}$. So if $\mu\left(C_{j}\left(B_{R}\right)\right) \neq 0$, then

$$
\begin{aligned}
\frac{\mu_{\mathcal{G}}\left(\Psi\left[C_{j}\left(U_{j}\right)\right]\right)}{\mu_{\mathcal{G}}\left(\Psi\left[C_{j}\left(B_{R}\right)\right]\right)} & =\frac{\mu_{\mathcal{G}}^{\prime}\left(\Psi\left[C_{j}\left(U_{j}\right)\right]\right)}{\mu_{\mathcal{G}}^{\prime}\left(\Psi\left[C_{j}\left(B_{R}\right)\right]\right)} \\
& =\frac{\mu\left[C_{j}\left(U_{j}\right)\right]}{\mu\left[C_{j}\left(B_{R}\right)\right]} .
\end{aligned}
$$

Also by definition of $C_{j}\left(U_{j}\right)$ and $\Psi$,

$$
\begin{aligned}
\mu_{\mathcal{G}}\left(\Psi\left[C_{j}\left(U_{j}\right)\right]\right) & =\mu_{\mathcal{G}}\left(\left\{g \in \mathcal{G} \mid \mathcal{O} \in g\left(U_{j} \cap \mathcal{F}_{j}\right)\right\}\right) \\
& =\mu_{\mathcal{G}}\left(\left\{g \in \mathcal{G} \mid g^{-1}(\mathcal{O}) \in U_{j} \cap \mathcal{F}_{j}\right\}\right) \\
& =\mu_{\mathcal{G}}\left(\left\{g \in \mathcal{G} \mid g(\mathcal{O}) \in U_{j} \cap \mathcal{F}_{j}\right\}\right) \\
& =\mu_{m}\left(U_{j} \cap \mathcal{F}_{j}\right) .
\end{aligned}
$$

The third equality holds because $\mu_{\mathcal{G}}$ is inversion invariant (i.e. $\mathcal{G}$ is unimodular); and the last equality holds since $\mu_{m}$ is the push-forward of $\mu_{\mathcal{G}}$ under the projection $\pi_{\mathcal{O}}: \mathcal{G} \rightarrow$ $\mathbb{H}^{m}$. Similarly, $\mu_{\mathcal{G}}\left(\Psi\left[C_{j}\left(B_{R}\right)\right]\right)=\mu_{m}\left(B_{R} \cap \mathcal{F}_{j}\right)$. Since both $U_{j}$ and $B_{j}$ are invariant under $G_{v_{j}}$,

$$
\frac{\mu_{m}\left(U_{j} \cap \mathcal{F}_{j}\right)}{\mu_{m}\left(B_{R} \cap \mathcal{F}_{j}\right)}=\frac{\mu_{m}\left(U_{j}\right)}{\mu_{m}\left(B_{R}\right)} .
$$

Equations (14)-(16) now show

$$
\begin{aligned}
\mu\left(C_{j}\left(U_{j}\right)\right) & =\mu\left(C_{j}\left(B_{R}\right)\right)\left(\frac{\mu_{\mathcal{G}}\left(\Psi\left[C_{j}\left(U_{j}\right)\right]\right)}{\mu_{\mathcal{G}}\left(\Psi\left[C_{j}\left(B_{R}\right)\right]\right)}\right) \\
& =\mu\left(C_{j}\left(B_{R}\right)\right)\left(\frac{\mu_{m}\left(U_{j} \cap \mathcal{F}_{j}\right)}{\mu_{m}\left(B_{R} \cap \mathcal{F}_{j}\right)}\right) \\
& =\mu\left(C_{j}\left(B_{R}\right)\right)\left(\frac{\mu_{m}\left(U_{j}\right)}{\mu_{m}\left(B_{R}\right)}\right) .
\end{aligned}
$$

Equation (9) is now proven. Equations (10) and (11) are obvious. To prove (12) it suffices to prove that $\left|\mu_{m}\left(B_{R}\right) / \mu_{m}\left(U_{j}\right)-\mu_{m}\left(B_{R}\right) / \mu_{m}\left(v_{j}\right)\right|<\varepsilon$. Since $v_{j}-N_{\delta}\left(\partial v_{j}\right) \subset U_{j}$ (by the discussion after the definition of $U_{j}$ ), the second condition on the choice of $\delta$ implies that $\left|\mu_{m}\left(B_{R}\right) / \mu_{m}\left(U_{j}\right)-\mu_{m}\left(B_{R}\right) / \mu_{m}\left(v_{j}\right)\right|<\varepsilon$ as required.

Equation (13) is due to the fifth condition on the choice of the collection $\left\{V_{j}\right\}_{j=1}^{n}$.

The following proves part of Theorem 2 .

Proposition 4. For any tight radius $R$, with associated tight-simplex packing $x$, the periodic measure $\mu_{x}$ is optimally dense. 
Proof. Theorem 1 in [Bo2] proves that the Voronoi cells in $x$ have the highest relative density among Voronoi cells of all packings in $S_{R}$. From this and Proposition 3 it follows that $\mu_{x}$ (and $x$ ) is optimally dense.

Propositions 1 and 3 show how the density of invariant measures can be understood locally. By contrast, the examples of Böröczky show that there are packings whose density cannot be well-defined from local structures.

\section{Proofs of the Theorems}

Proof of Theorem 1. $\quad F_{\mathcal{O}}$ is upper semicontinuous, and it is easy to construct a decreasing sequence $F_{j}$ of continuous real-valued functions on $S_{R}$ which converge pointwise to $F_{\mathcal{O}}$. Choose a sequence $\mu^{k} \in \mathcal{M}_{I}^{\mathrm{e}}(R)$ such that $D\left(\mu^{k}\right)=\int_{S_{R}} F_{\mathcal{O}} d \mu^{k} \rightarrow D(R)$ as $k \rightarrow \infty$, and, using the compactness of $\mathcal{M}_{I}(R)$, assume without loss of generality that $\mu^{k}$ converges to some $\mu^{\infty} \in \mathcal{M}_{I}(R)$. Then $\int_{S_{R}} F_{j} d \mu^{k} \rightarrow \int_{S_{R}} F_{j} d \mu^{\infty}$ as $k \rightarrow \infty$, and $\int_{S_{R}} F_{j} d \mu^{\infty} \searrow D\left(\mu^{\infty}\right)$ as $j \rightarrow \infty$. Since $\int_{S_{R}} F_{j} d \mu^{k} \geq D\left(\mu^{k}\right)$ and $D\left(\mu^{k}\right) \rightarrow D(R)$ as $k \rightarrow \infty, D\left(\mu^{\infty}\right) \geq D(R)$. From the Krein-Milman theorem there exists $\tilde{\mu} \in \mathcal{M}_{I}^{\mathrm{e}}(R)$ for which $D(\tilde{\mu})=\int_{S_{R}} F_{\mathcal{O}} d \tilde{\mu} \geq D\left(\mu^{\infty}\right)$, and thus $D(\tilde{\mu}) \geq D(R)$. However, then from the definition of $D(R), D(\tilde{\mu})=D(R)$.

We now show that there are optimally dense packings in the support $\sigma(\tilde{\mu})$ of $\tilde{\mu}$. Choose a countable dense subset $A$ in $\sigma(\tilde{\mu})$. Let $C$ be the collection of all balls whose center lies in $A$ and whose radius is $1 / n$ for some positive integer $n$. For each of the balls $c \in C$ there is a subset of $\sigma(\tilde{\mu})$, of full $\tilde{\mu}$-measure, whose orbit intersects $c$, as we see by applying Nevo's ergodic theorem [NeS, Theorem 3] to the indicator function of $c$. It follows that there is a set of full $\tilde{\mu}$-measure of points, each of whose orbit intersects every ball in $C$. The closure of any such orbit must therefore contain $A$. Since $A$ is dense in $\sigma(\tilde{\mu})$, any such orbit must also be dense.

To prove Theorem 2, we use four preliminary lemmas and the following proposition, together with the notion of the girth of a subgroup $G<\mathcal{G}$, which is defined to be $\inf _{a \in \mathbb{H}^{m}} \inf _{g \in G-\{e\}} d(a, g a)$.

Proposition 5. If $G<\mathcal{G}$ is discrete and cocompact and $T>0$, then there exists a finite index subgroup $H<G$ such that $H$ is torsion-free and the girth of $H$ is greater than $T$.

Proof. This is established in the proof of Theorem 4.1 in [FKK].

The following result is stated, without proof, at the bottom of page 142 of [FKK], using the following definition. A packing $x$ is called "completely saturated" if whichever $N$ balls are removed from $x, N+1$ may not be added to the remainder and still produce a packing. Recall that if $x$ is a periodic packing in $S_{R}$, then there is a canonical measure $\mu_{x} \in M_{I}^{\mathrm{e}}(R)$ whose support is equal to the orbit of $x$ in $S_{R}$. For $x \in S_{R}$, we say that the radius of $x$ is $R$ and denote it by $\mathcal{R}(x)=R$. 
Lemma 1. Suppose $x$ is a periodic packing. If $\mu_{x}$ is optimally dense, then $x$ is completely saturated.

Proof. Suppose $x$ is not completely saturated. Then there exists a packing $x^{\prime}$ with the following three properties. First, $\mathcal{R}\left(x^{\prime}\right)=\mathcal{R}(x)$. Second, there is some $q \in C_{x^{\prime}}, q \notin C_{x}$, and a number $R$ such that if $c \in C_{x^{\prime}}$ is not contained in the ball of radius $R$ with center $q$, then $c \in C_{x}$. Also, conversely, if $c \in C_{x}$ and is not contained in the ball of radius $R$ with center $q$, then $c \in C_{x^{\prime}}$. Third, the number of centers of $x^{\prime}$ contained in the ball of radius $R$ centered at $q$ is greater than the number of centers of $x$ contained in the ball of radius $R$ centered at $q$. Let $G_{0} \subset \Gamma_{x}$ be a finite index subgroup of $\Gamma_{x}$ that is torsion-free and has girth greater than $R$. Then there exists a fundamental domain $\mathcal{F}$ for $G_{0}$ containing the ball of radius $R$ centered at $q$. Let $x^{\prime \prime}=\bigcup_{g \in G_{0}} g\left(x^{\prime} \cap \mathcal{F}\right)$. Since $x^{\prime}$ is the same as $x$ outside the ball of radius $R$ centered at $q$, it follows that $x^{\prime \prime}$ is a packing. Since $\mathcal{F}$ is a fundamental domain for $G_{0}$ and the relative volume of $x^{\prime \prime}$ in $\mathcal{F}$ is greater than the relative volume of $x$ in $\mathcal{F}$, it follows from Proposition 1 that $\mu_{x}$ is not optimally dense.

Let $q \in \mathbb{H}^{m}$. For $a \in \mathbb{H}^{m}$ such that $a \neq q$ and $s>0$ define $a_{q}(s)$ to be the point on the ray from $q$ to $a$ such that $d\left(q, a_{q}(s)\right)=d(q, a)+s$.

Lemma 2. Let $q \in \mathbb{H}^{m}$. If $a, b$ are any distinct points of $\mathbb{H}^{m}$ but neither equal to $q$ and $s>0$, then $d(a, b) \leq d\left(a_{q}(s), b_{q}(s)\right)$.

Proof. Let $a q b$ denote the acute angle between $a q$ and $q b$. Define a function $H$ by

$$
H(y, z, s)=\cosh (y+s) \cosh (z+s)-\cos (a q b) \sinh (y+s) \sinh (z+s) .
$$

Then, by the law of cosines (see [Rat]), $H(d(a, q), d(b, q), s)=\cosh \left[d\left(a_{q}(s), b_{q}(s)\right)\right]$. So it suffices to show that the derivative of $H$ at $(y, z, s)$ with respect to $s$ is positive whenever all the variables $y, z$ and $s$ are positive. So we compute:

$$
\begin{aligned}
d H / d s= & \sinh (y+s) \cosh (z+s)+\cosh (y+s) \sinh (z+s) \\
& -\cos (a q b)[\cosh (y+s) \sinh (z+s)+\sinh (y+s) \cosh (z+s)] \\
= & (1-\cos (a q b))[\cosh (y+s) \sinh (z+s)+\sinh (y+s) \cosh (z+s)] \\
\geq & 0 . \quad \square
\end{aligned}
$$

Lemma 3. Suppose $x$ is a packing for which there exists $t>0$ such that the distance between any two centers of $x$ is greater than or equal to $2 \mathcal{R}(x)+t$. Then $x$ is not completely saturated.

Proof. The idea behind the proof is that by moving a finite number of balls away from a point $q \notin C_{x}$, there will be space enough to place a new ball with center at $q$. 
Let $k$ be the integer such that $k t>2 \mathcal{R}(x) \geq(k-1) t$ and let $R=k(2 \mathcal{R}(x)+t)$. We define a function $f$ on $C_{x}$ as follows:

(i) If $d(c, q) \geq R$, then let $f(c)=c=c_{q}(0)$.

(ii) Otherwise there is a $j$ such that $0<j \leq k$ and $R-2(j-1)(\mathcal{R}(x)+t)>$ $d(q, c) \geq R-2 j(\mathcal{R}(x)+t)$. In this case, define $f(c)=c_{q}(j t)$.

Let $C^{\prime}$ be the union of the point $q$ and the image of $C_{x}$ under $f$. From the definitions, it is clear that $C^{\prime}$ differs from $C_{x}$ in only a finite number of points. Hence once we show that balls of radius $\mathcal{R}(x)$ centered at points of $C^{\prime}$ do not overlap, it follows that $x$ is not completely saturated.

Note that if $c \in C_{x}$ and $f(c)=c_{q}(j t)$, then

$$
\begin{aligned}
d(f(c), q) & =d\left(c_{q}(j t), q\right) \\
& =d(c, q)+j t \\
& \geq R-2 j[\mathcal{R}(x)+t]+j t \\
& =(k-j)[2 \mathcal{R}(x)+t] .
\end{aligned}
$$

Hence if the ball of radius $\mathcal{R}(x)$ centered at $f(c)$ overlaps the ball of radius $\mathcal{R}(x)$ centered at $q$, then $f(c)=c_{q}(k t)$. However, this implies that $d(q, f(c))>k t>2 \mathcal{R}(x)$, a contradiction. Hence the balls of radius $\mathcal{R}(x)$ centered at $q$ and $f(c)$ do not overlap for any $c \in C_{x}$.

Now assume for a contradiction that there exist two distinct centers $c$ and $c^{\prime}$ in $C_{x}$ with $d\left(f(c), f\left(c^{\prime}\right)\right)<2 \mathcal{R}(x)$. If $d(c, q) \geq R$ and $d\left(c^{\prime}, q\right) \geq R$, then $f(c)=c$ and $f\left(c^{\prime}\right)=c^{\prime}$ so $d\left(f(c), f\left(c^{\prime}\right)\right)=d\left(c, c^{\prime}\right)>2 \mathcal{R}(x)$, a contradiction. So we may assume that $d\left(q, c^{\prime}\right)<R$. Suppose that $f(c)=c_{q}(j t)$ and $f\left(c^{\prime}\right)=c_{q}^{\prime}\left(j^{\prime} t\right)$. Note by the distance inequality, the assumption that $d\left(f(c), f\left(c^{\prime}\right)\right)<2 \mathcal{R}(x)$ and the definition of $f$,

$$
\begin{aligned}
d(f(c), q) & \leq d\left(f(c), f\left(c^{\prime}\right)\right)+d\left(f\left(c^{\prime}\right), q\right) \\
& <2 \mathcal{R}(x)+d\left(c^{\prime}, q\right)+j^{\prime} t \\
& <2 \mathcal{R}(x)+R-2\left(j^{\prime}-1\right)[\mathcal{R}(x)+t]+j^{\prime} t .
\end{aligned}
$$

Next by definition of $R$,

$$
2 \mathcal{R}(x)+R-2\left(j^{\prime}-1\right)[\mathcal{R}(x)+t]+j^{\prime} t=\left(k-j^{\prime}+2\right)[2 \mathcal{R}(x)+t] .
$$

The previous equations (20)-(22) now imply that

$$
0<\left(j-j^{\prime}+2\right)[2 \mathcal{R}(x)+t] .
$$

So

$$
j^{\prime}<j+2 .
$$

If $j=0$, then $j^{\prime}=1$. Otherwise $d(q, c)<R$ and so, by symmetry,

$$
j<j^{\prime}+2 .
$$


In any case, we may assume that either $j^{\prime}=j$ or $j^{\prime}=j+1$. So Lemma 2 shows that

$$
\begin{aligned}
2 \mathcal{R}(x)+t & \leq d\left(c, c^{\prime}\right) \\
& \leq d\left(c_{q}(j t), c_{q}^{\prime}(j t)\right) \\
& =d\left(f(c), c_{q}^{\prime}(j t)\right) \\
& \leq d\left(f(c), c_{q}^{\prime}\left(j^{\prime} t\right)\right)+d\left(c_{q}^{\prime}\left(j^{\prime} t\right), c_{q}^{\prime}(j t)\right) \\
& =d\left(f(c), f\left(c^{\prime}\right)\right)+d\left(c_{q}^{\prime}\left(j^{\prime} t\right), c_{q}^{\prime}(j t)\right) \\
& <2 \mathcal{R}(x)+t .
\end{aligned}
$$

This contradiction finishes the lemma.

For a packing $x$, let $K(x)$ be the $m$-complex underlying the Delone cell decomposition of $x$. In other words, the vertex set of $K(x)$ is equal to the set of ball centers of $x$, an edge exists between vertices $v_{1}$ and $v_{2}$ if and only an edge of a Delone cell connects the corresponding centers in $x$, and so on. Let $\operatorname{Aut}(x)$ denote the automorphism group of $K(x)$, i.e. the group of bijective maps from $K(x)$ to $K(x)$ that preserve its structure as an $m$-complex.

Lemma 4. If $x_{0}$ and $x_{1}$ are periodic packings, $K\left(x_{0}\right)$ and $K\left(x_{1}\right)$ are isomorphic as $m$-complexes, and $\mathcal{R}\left(x_{0}\right)<\mathcal{R}\left(x_{1}\right)$, then $\mu_{x_{0}}$ is not optimally dense.

Proof. Let $x_{2}$ be the packing such that $C_{x_{2}}=C_{x_{1}}$ and $\mathcal{R}\left(x_{2}\right)=\mathcal{R}\left(x_{0}\right)$. Intuitively, $x_{2}$ is formed from $x_{1}$ by shrinking the radius of the balls to $\mathcal{R}\left(x_{0}\right)$. Let $t=2 \mathcal{R}\left(x_{1}\right)-2 \mathcal{R}\left(x_{0}\right)>$ 0 . By Lemma $3, x_{2}$ is not completely saturated. By Lemma $1, \mu_{x_{2}}$ is not optimally dense. We show that $D\left(\mu_{x_{2}}\right)=D\left(\mu_{x_{0}}\right)$. Given this it follows that $\mu_{x_{0}}$ is not optimally dense, which proves the lemma.

Note that $K\left(x_{1}\right)=K\left(x_{2}\right)$, so $K\left(x_{0}\right)$ and $K\left(x_{2}\right)$ are isomorphic as $m$-complexes. Hence there exists a homeomorphism $\Phi: \mathbb{H}^{m} \rightarrow \mathbb{H}^{m}$ such that $\Phi$ takes the $k$-cells of $K\left(x_{0}\right)$ to the $k$-cells of $K\left(x_{2}\right)$ for $0 \leq k \leq m$ and vice versa. Note that $\Phi$ induces an isomorphism $\Phi_{*}$ from $\operatorname{Aut}\left(x_{0}\right)$ to $\operatorname{Aut}\left(x_{2}\right)$ by $\Phi_{*}(\alpha)(u)=\Phi \alpha \Phi^{-1}(u)$ for any $\alpha \in$ $\operatorname{Aut}\left(x_{2}\right)$ and $u \in K\left(x_{2}\right)$. Also there are natural (injective) inclusion homeomorphisms $i_{j}: \Gamma_{x_{j}} \rightarrow \operatorname{Aut}\left(x_{j}\right)$ for $j=0$, 2. Since $\Gamma_{x_{j}}$ is cocompact (for $\left.j=0,2\right), i_{j}\left(\Gamma_{x_{j}}\right)$ has finite index in $\operatorname{Aut}\left(x_{j}\right)$. Let $\Gamma_{t}$ be a finite-index torsion-free subgroup of $\Gamma_{x_{0}}$. The group $\hat{H}_{2} \equiv \Phi_{*} i_{0}\left(\Gamma_{t}\right) \cap i_{2}\left(\Gamma_{x_{2}}\right)$ has finite index in $\operatorname{Aut}\left(x_{2}\right)$. So $\hat{H}_{0} \equiv \Phi_{*}^{-1}\left(H_{2}\right)$ has finite index in $\operatorname{Aut}\left(x_{0}\right)$. So $H_{j}=i_{j}^{-1}\left(\hat{H}_{j}\right)$ for $j=0,2$ has finite index in $\Gamma_{x_{j}}$. Note both $H_{0}$ and $H_{2}$ are torsion free. The Mostow Rigidity Theorem states that if the dimension of $\mathbb{H}^{m}$ is greater than 2 and $H_{0}$ and $H_{2}$ are isomorphic, discrete, cofinite, torsion-free subgroups of $\mathcal{G}$, then any isomorphism from $H_{0}$ and $H_{1}$ can be realized by an isometry from $\mathbb{H}^{m} / H_{0}$ to $\mathbb{H}^{m} / H_{2}$ (see [Kap]). In particular, $\mathbb{H}^{m} / H_{0}$ and $\mathbb{H}^{m} / H_{2}$ have the same volume. When the dimension of $\mathbb{H}^{m}$ equals 2, the Gauss-Bonnet theorem implies that $\mathbb{H}^{m} / H_{0}$ and $\mathbb{H}^{m} / H_{2}$ have the same area. By the homeomorphism $\Phi, K\left(x_{0}\right) / H_{0}$ has the same number of vertices as $K\left(x_{1}\right) / H_{1}$. Hence $x_{0} / H_{0}$ has the same number of balls as $x_{2} / H_{2}$. Proposition 1 now implies that $D\left(\mu_{x_{0}}\right)=D\left(\mu_{x_{2}}\right)$. 
Proof of Theorem 2. Lemma 4 implies that the set of all radii that admit an optimally dense periodic measure injects into the set of finite $m$-complexes (by a map that sends $\mu_{x}$ to $K(x) / H$ where $H$ is some cocompact torsion free subgroup of $\Gamma_{x}$ ). The later set is countable so the first part of Theorem 2 follows immediately. The second part was proven by Proposition 4 .

\section{Conclusion}

For any packing of balls of fixed radius in a Euclidean space it is possible to define an "upper density" as the limit supremum, of the relative density of the balls in a system of spheres expanding about any fixed point in space, as the radius of the spheres goes to infinity. Then an optimal "packing density" can be defined as the supremum of the upper densities of all packings [FeK], [CoS], [Rog].

As we discussed in the Introduction, the situation seems intrinsically more complicated in hyperbolic space, with the example of Böröczky representing one challenge. The definition we propose for optimally dense measures and packings circumvents some of the difficulties of more direct approaches because the troublesome packings (like Börözcky's) make up a set of measure zero for every invariant measure on the space of packings. We justify our definition of the optimal density of packings in three ways: (1) by its relation to the limit of the density in expanding compact subregions of packings, using the ergodic theorem of Nevo; (2) by its agreement with the average density in Voronoi, and Delone, cells; (3) by its agreement, for periodic packings, with density in a fundamental domain.

In more detail, each invariant probability measure $\mu$ on the space $S_{R}$ of all packings allowed us, through Nevo's ergodic theorem, to avoid naturally the ( $\mu$-negligible) set of packings for which density is problematic, and indeed show that over the remaining packings the average density $D(\mu)$ not only makes sense but agrees with the average relative density with respect to Voronoi cells or Delone cells. We then defined optimal density by taking the supremum over invariant measures, and proved that: for the countable number of "tight" radii, the obvious packings, of high symmetry, are optimally dense; and that for all but countably many radii, optimally dense packings must have low symmetry. Further progress should come when the first specific example of a low-symmetry optimum is analyzed.

For many years now there have been studies of "aperiodic tilings" in Euclidean spaces, particularly the plane. Such tilings correspond to the situation in which one has a finite set of polygons (or polyhedra), congruent copies of which can tile space but never by a "periodic" tiling, that is, one with the symmetry group cocompact in the Euclidean group [Ra2]). Generalizations to spaces other than the Euclidean plane have not been obvious; see for instance [Ra1] and [Moz].

This paper grew from an attempt to understand the significance of recent constructions of aperiodic tilings in the hyperbolic plane; see for instance [BIW], [Moz], [MaM] and [G] (based in part on [P]). We feel it is appropriate and useful to think of aperiodic tilings (in Euclidean or hyperbolic space) as optimally dense packings, which led us to the above study of the "simpler" situation of ball packings in hyperbolic space. This study suggests that an important element in dealing with optimally dense packings, at least in hyperbolic 
space, is the use of Borel probability measures, on a relevant space of packings, invariant under the congruence group. (Such measures have been used extensively to analyze aperiodic tilings in Euclidean spaces [Ra2], but to study symmetry, not density.) Unlike the situation for Euclidean space, in hyperbolic space there is a complication from the fact that when the congruence group acts on a compact metric space (such as a special space of packings) there may not exist an invariant probability measure on that space. (A well known example is the natural action of $P S L_{2}(\mathbb{R})$ on the boundary of the hyperbolic plane.) This suggests that it is inappropriate to attempt to define a density for some packings, for instance one for which there does not exist an invariant measure on the closure of its orbit under all rigid motions. In effect, the use of invariant measures for defining optimal density brings with it a restriction, on the class of packings which can be considered optimally dense, akin to the restriction of measurability shown to be necessary, in fundamental approaches to volume using equidecomposability, by the Banach-Tarski paradox [W].

\section{Acknowledgment}

We are very grateful to Oded Schramm for pointing us to $[\mathrm{BeS}]$ and its use in defining density in the hyperbolic plane.

\section{References}

[Bea] A. Beardon, The Geometry of Discrete Groups, Springer-Verlag, New York, 1983.

[BeS] I. Benjamini and O. Schramm, Percolation in the hyperbolic plane, J. Amer. Math. Soc. 14 (2001), 487-507.

[BIW] J. Block and S. Weinberger, Aperiodic tilings, scalar curvature, and amenability of spaces, J. Amer. Math. Soc. 5 (1992), 907-918.

[Bo1] K. Böröczky, Sphere packing in the hyperbolic plane (in Hungarian), Mat. Lapok 25 (1974), 265-306.

[Bo2] K. Böröczky, Packing of spheres in spaces of constant curvature, Acta Math. Acad. Sci. Hungar. 32 (1978), 243-261.

[Bow] L. Bowen, Sphere packing in the hyperbolic plane, Math. Phys. Electron. J. 6(6) (2000), 1-10.

[CoS] J.H. Conway and N.J.A. Sloane, Sphere Packings, Lattices and Codes, third edition, Springer-Verlag, New York, 1999.

[Fej] L. Fejes Tóth, Regular Figures, Macmillan, New York, 1964.

[FeK] G. Fejes Tóth and W. Kuperberg, Packing and covering with convex sets, chapter 3.3, pp. 799-860, in Vol B of Handbook of Convex Geometry, ed. P. Gruber and J. Wills, North-Holland, Amsterdam, 1993.

[FKK] G. Fejes Tóth, G. Kuperberg and W. Kuperberg, Highly saturated packings and reduced coverings, Monatsh. Math. 125 (1998), 127-145.

[G] C. Goodman-Strauss, A strongly aperiodic set of tiles in the hyperbolic plane, preprint, Univ. Arkansas, 2000.

[H] T. Hales, preprints available from http://www.math.lsa.umich.edu/hales/countdown/

[Kap] M. Kapovich, Hyperbolic Manifolds and Discrete Groups, Birkhäuser, Boston, MA, 2001.

[Kat] S. Katok, Fuchsian Groups, University of Chicago Press, Chicago, IL, 1992.

[MaM] G.A. Margulis and S. Mozes, Aperiodic tilings of the hyperbolic plane by convex polygons, Israel J. Math. 107 (1998), 319-332.

[Moz] S. Mozes, Aperiodic tilings, Invent. Math. 128 (1997), 603-611.

[Ne] A. Nevo, Pointwise ergodic theorems for radial averages on simple Lie groups I, Duke Math. J. 76 (1994), 113-140. 
[NeS] A. Nevo and E. Stein, Analogs of Weiner's ergodic theorems for semisimple groups, I, Ann. of Math. 145 (1997), 565-595.

[P] R. Penrose, Pentaplexy, Eureka 39 (1978), 16-22.

[Ra1] C. Radin, Aperiodic tilings in higher dimensions, Proc. Amer. Math. Soc. 123 (1995), 3543-3548.

[Ra2] C. Radin, Miles of Tiles, Student Mathematical Library, Vol. 1, American Mathematical Society, Providence, RI, 1999.

[Rat] J. Ratcliffe, Foundations of Hyperbolic Manifolds, Graduate Text in Mathematics, Vol. 149, SpringerVerlag, New York, 1994.

[RaW] C. Radin and M. Wolff, Space tilings and local isomorphism, Geom. Dedicata 42 (1992), 355-360.

[Rog] C.A. Rogers, Packing and Covering, University Press, Cambridge, 1964.

[V] E.B. Vinberg, ed., Geometry II, Encyclopedia of Mathematical Sciences, Vol. 29, Springer-Verlag, Berlin, 1993.

[W] S. Wagon, The Banach-Tarski Paradox, Cambridge University Press, Cambridge, 1985.

Received November 15, 2000, and in revised form October 5, 2001, and April 15, 2002.

Online publication October 29, 2002. 